\title{
Fiber Optical Parametric Amplification of Optical Combs for Enhanced Performance and Functionality
}

\author{
Joseph Kakande, Periklis Petropoulos and David J. Richardson \\ Optoelectronics Research Centre, University of Southampton, Southampton, SO17 1 BJ, United Kingdom \\ Authore-mail address: jkk@orc.soton.ac.uk
}

\begin{abstract}
We demonstrate the inherent suitability of fiber optical parametric amplifiers (FOPAs) to the amplification of optical combs, combining gain, spectral shaping, frequency translation, effective spectral broadening and low noise amplification.

OCIS codes: (060.2320) Fiber optics amplifiers and oscillators, (190.4410) Nonlinear optics, parametric processes
\end{abstract}

\section{Introduction}

The performance of FOPAs today has been significantly improved by the advances in both highly nonlinear fiber (HNLF), which acts as the gain medium, as well as improved pump sources offering high coherence and high power (especially when combined with pump pre-amplification in a doped fiber or Raman amplifier). Improvements in the achievable tolerance in the distributed physical characteristics of HNLF allow phase matching over long distances (on the order of hundreds of meters), allowing much broader gain bandwidths than doped fiber amplifiers (DFAs), as well as engineerable operating windows across the IR spectrum [1]. High coherence pump sources also enable phase sensitive FOPA operation, allowing both a noise figure below the $3 \mathrm{~dB}$ quantum limit (when operated as linear devices) [2], and the possibility of sophisticated signal processing functions such as phase regeneration (when designed to be nonlinear) [3]. Finally, by boosting pump power sufficiently FOPAs can provide very high signal gains with over $40 \mathrm{~dB}$ demonstrated [1].

Despite all these desirable characteristics, FOPAs today still do not at present offer an economically competitive alternative to DFAs, particularly for telecoms applications. This is due to a combination of many factors - DFAs are low cost and proven, have high quantum efficiency, are robust, have low susceptibility to stimulated Brillouin scattering (SBS), and conveniently already cover the low loss wavelength region in silica which is of most interest for current long haul optical communications. In addition, the very same nonlinearity that provides the gain mechanism in FOPAs can also cause deleterious nonlinear crosstalk between multiple channels during amplification [4]. While many of these issues are being addressed by the fabrication of fibers with novel structure and composition [5], we here present the linear FOPA in a different light, as a device allowing multiple functionalities inherently suitable for the amplification of optical combs, particularly those derived from resonant cavity + modulator structures.

Optical combs provide an absolute reference in either the spectral or time domains, and as such have a vast range of applications in physical and life sciences [6]. In most comb generators, energy is transferred from a seed beam to a large number of frequency detuned comb lines, and energy conservation dictates that the individual comb lines consequently have low power and low optical signal to noise ratio (OSNR), which is further compromised whenever attempts are made to transmit the comb(s) through lossy media. In addition, some combs, particularly those generated from resonant cavity structures [7] inherently have a spectral roll-off, with less power as the comb line detuning from seed carrier increases. Finally, the requirement to act as a reference means that combs are often required in spectral areas outside the telecom window. As a result, an ideal amplifier for optical combs should combine low noise with gain shaping (i.e. un-equalised gain) and a frequency translation capability. Here, we demonstrate how FOPAs allow all these features to be attained, and attempt to objectively assess their ultimate suitability for this application.

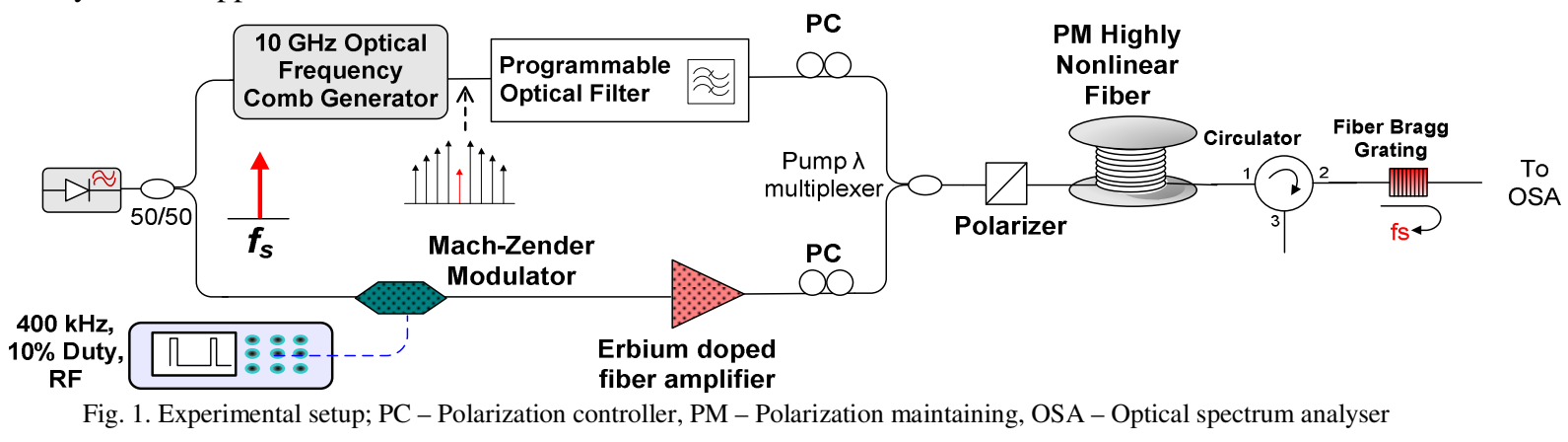




\section{Implementation and setup}

The setup is shown in Fig. 1. A narrow linewidth CW signal at $1550.92 \mathrm{~nm}$ seeded an optical frequency comb generator (OFCG) from Optocomb Inc [7]. The OFCG comprises a phase modulator inside a high finesse Fabry Perot cavity driven with an internal $10 \mathrm{GHz}$ RF clock. As shown in Fig. 2a, the output comb has a triangular spectral roll off, as well as a high insertion loss of approximately $30 \mathrm{~dB}$. The comb was then passed through a reconfigurable filter (Finisar Waveshaper 4000E) which was used to switch between amplifying the full spectrum or only half of it, the necessity of which is discussed shortly. For a parametric comb amplifier, it is essential that the pump beam is locked in frequency to the comb, and for a comb spacing of $\Delta \mathrm{f}$, at least a whole multiple of $\Delta \mathrm{f}$ away from the nearest comb line. In addition, the pump linewidth should be no more than that of the comb lines. This requirement is easily satisfied by injection locking a semiconductor laser to one of the comb lines, a technique we have previously demonstrated for phase regeneration applications [8]. For simplicity though, we chose here to tap the same laser as the comb seed. The comb and pump were combined with a $100 \mathrm{GHz}$ add-drop multiplexer at the pump wavelength. This suppressed comb lines within $\pm 50 \mathrm{GHz}$ of the pump, although this can be reduced by using either a narrower multiplexer or a narrow grating. Polarization controllers and a polarizer ensured the comb and pump were copolarized, and they were then launched into the fast axis of a 493m PM HNLF (the FOPA), with fast axis zero dispersion wavelength (ZDW) $1544 \mathrm{~nm}$, slope $0.029 \mathrm{ps} / \mathrm{nm} / \mathrm{km}^{2}$ and nonlinear coefficient $10.7 / \mathrm{W} / \mathrm{Km}$ (OFS Fitel, Denmark). The SBS threshold of this fiber was around $18 \mathrm{dBm}$, which was significantly lower than the desired launch pump power. While SBS suppression can be derived without degrading the effective pump linewidth in the parametric process by a number of techniques (including using dual counter-phased pumps, longitudinal straining, aluminium oxide core doping [5], and mid-span isolation amongst others), we opted for ease of implementation given the low dispersion fibers available to us, to use in this instance a quasi-CW pump gated in an amplitude modulator at $400 \mathrm{kHz}$ with a $10 \%$ duty cycle, effectively restricting the nonlinear interaction to approximately $100 \mathrm{~m}$. The comb power at the input to the FOPA was $-40 \mathrm{dBm}$. The FOPA was operable in both phase-insensitive (PI) mode, in which case only the half of the comb with wavelength shorter than that of the pump was coupled into the FOPA, as well as in phase-sensitive (PS) mode, in which the pump is located precisely in the middle of the symmetric comb spectrum. In PS mode, the gain experienced by each comb line depends on the instantaneous phase relationship between that comb line, the pump, and the comb line at the same spectral detuning but on the other side of the pump in frequency. To maintain the PS-FOPA at optimal phase a feedback loop monitoring the PS-FOPA output is normally required to compensate for relative phase drifts resulting from thermal and acoustic pickup. If $n$ uncorrelated channels (such as multiple dispersed comb lines) are being amplified, $n$ feedback loops as channels are required, an undesirable level of complexity. Instead, we set the OSA to perform multiple spectral sweeps of the output of the unlocked PS-FOPA while in maximum-hold mode. The resulting spectrum rapidly converges to that which would be measured if the pump and all the comb lines were phase locked at the PS maximum gain value. Conversely, operating the OSA in minimum-hold mode provides the PS-minimum spectrum.

\section{Results and discussion}

The spectrum at the FOPA output with the pump off is shown in Fig. 2a. The OFCG provides a comb with $-10 \mathrm{~dB} /$ $\mathrm{THz}$ spectral roll-off. The pump power was adjusted to ensure that the non-flat gain spectrum of the FOPA cancelled out the spectral roll-off of the input comb, i.e. obtain a $+10 \mathrm{~dB} / \mathrm{THz}$ gain shape. Positive gain slopes are easiest obtained by placing the pump in the anomalous dispersion regime, and the magnitude of the slope can be controlled by the pump power [4]. We show output spectra in Figs. 2b and 2c. Note that the region between 1550 and $1551 \mathrm{~nm}$ should ignored as this is where the pump is filtered with some energy remaining. In Fig. $2 \mathrm{~b}$, it can be seen that we obtain under $1.5 \mathrm{~dB}$ variation over $8 \mathrm{~nm}$ (approx. $1 \mathrm{THz}$ ). This is best observed by viewing the right side of the spectrum (wavelengths $>1551 \mathrm{~nm}$ ). The apparent deviation from this behaviour for short wavelengths is only an artifact of the $10 \%$ gating of the pump at the input with the OSA averaging measured power over both pump-on and pump-off cycles. We measured data for average pump powers of $23 \mathrm{dBm}$ and $26 \mathrm{dBm}$, corresponding to $33 \mathrm{dBm}$ and $36 \mathrm{dBm}$ pump peak powers approximately. In Fig. 3a we calculate the single sided PI gains at 33 and $36 \mathrm{dBm}$ peak pump power and reveal maximum gains of 19 and $33 \mathrm{~dB}$ respectively. The slope of the curve at $33 \mathrm{dBm}$ is very close to the $+10 \mathrm{~dB} / \mathrm{THz}$ target. In addition, in PI mode, the generation of idlers of the input comb lines means that the output spectrum is twice the width of the input, an important benefit. In Fig. $3 b$, we show the output spectrum in the absence of an input comb. The noise present on the pump beam and that generated in the HNLF is amplified, and this limits the output comb OSNR observable in Figs. 2b and 2c. Its impact would obviously be less critical for higher input signal powers. In Fig. 3c, we compare a narrow section of the spectrum when operated in PI and PS modes. The PS output shows both higher power and higher OSNR, as would be expected [2]. Within the comb section shown in Fig 3c, the maximum observed PS advantage is $4 \mathrm{~dB}$ (rightmost comb line). In simplistic terms, this $4 \mathrm{~dB}$ advantage of this particular PS-FOPA should be observed when benchmarked against any PI amplifier, such as a DFA. 

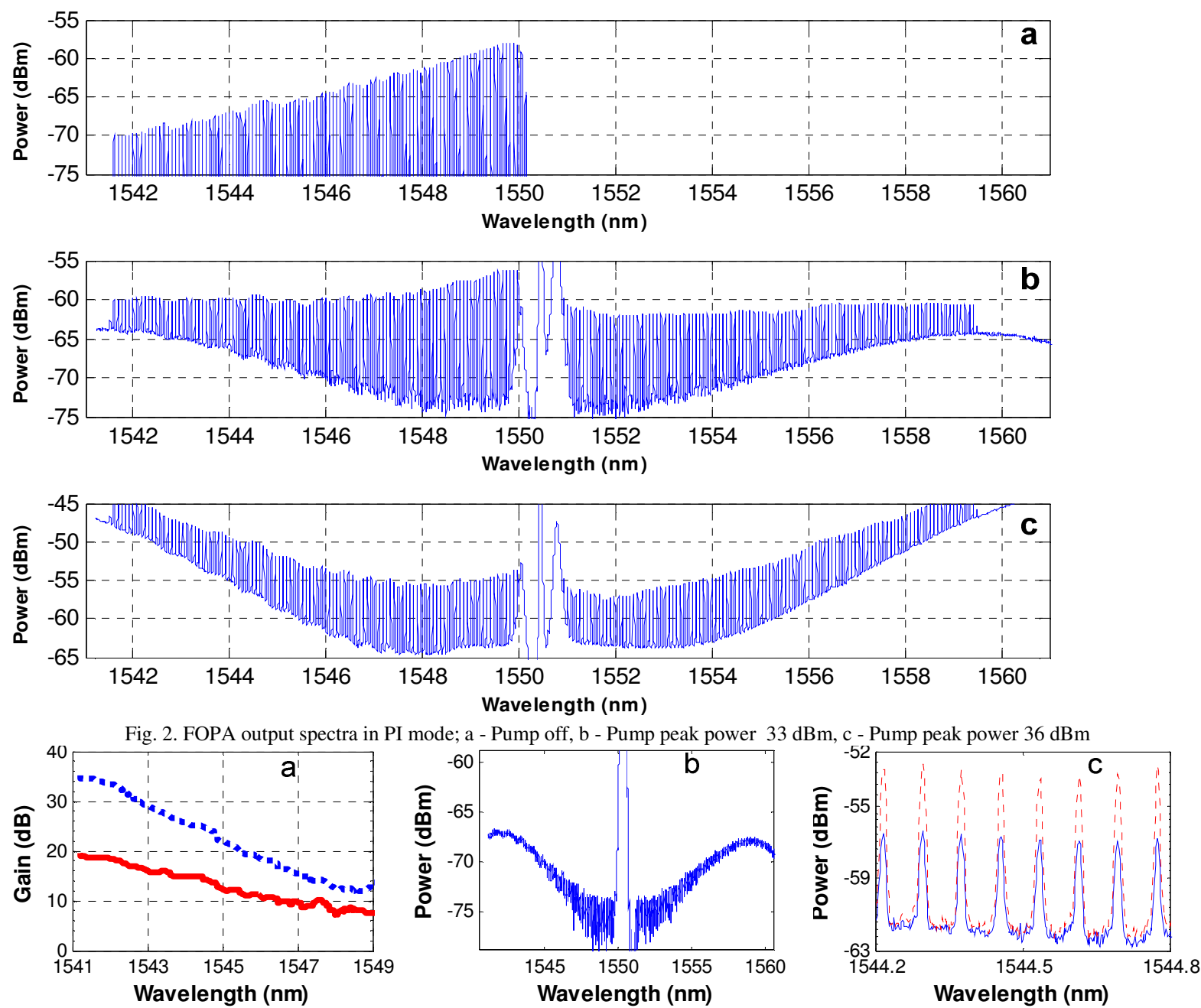

Fig. 3. a - Single sided PI gain curves for $33 \mathrm{dBm}$ peak power (solid line) and $36 \mathrm{dBm}$ (dotted line), $\mathrm{b}$ - output spectrum at $33 \mathrm{dBm}$ peak power in absence of input signal, $\mathrm{c}-$ zoom in of comb lines amplified in PI (solid) and PS (dotted) showing a PS noise figure enhancement $>3 \mathrm{~dB}$.

\section{Conclusions}

We have highlighted the suitability of FOPAs for the amplification of optical combs. The wavelength dependent gain of FOPAs pumped in the anomalous regime can be used for comb flattening. FOPAs can deliver suitably high gain (up to $33 \mathrm{~dB}$ shown here) and when operated in PS mode, superior noise performance with more than a $3 \mathrm{~dB}$ noise figure enhancement over a PI amplifier. Finally, when operated in PI mode the output spectrum is twice the width of the input spectrum, extending the spectral reach of the comb.

\section{Acknowledgement}

We thank OFS Denmark for supplying the PM-HNLF. This research has received funding from the European Communities Seventh Framework Programme FP/2007-2013 under grant agreement 224547 (PHASORS).

\section{References}

[1] K. K. Y. Wong, M. E. Marhic, G. Kalogerakis et al., "Fiber optical parametric amplifier and wavelength converter with record 360 nm gain bandwidth and $50 \mathrm{~dB}$ signal gain." p. 2 pp.

[2] T. Zhi, et al., "Measurement of sub-1 dB noise figure in a non-degenerate cascaded phase-sensitive fibre parametric amplifier." pp. 1-2.

[3] R. Slavik, F. Parmigiani, J. Kakande et al., "All-optical phase and amplitude regenerator for next-generation telecommunications systems," Nat Photon, vol. 4, no. 10, pp. 690-695, 2010.

[4] M. E. Marhic, "Fiber Optical Parametric Amplifiers, Oscillators and Related Devices," 2007.

[5] L. Grüner-Nielsen, S. Herstrøm, S. Dasgupta et al., "Silica-Based Highly Nonlinear Fibers with a High SBS Threshold," IEEE Photonics Society 2011 Winter Topical meeting, pp. Paper MD4.2 2011.

T. Udem, R. Holzwarth, and T. W. Hansch, "Optical frequency metrology," Nature, vol. 416, no. 6877, pp. 233-237, 2002.

[7] M. Kourogi, K. Nakagawa, and M. Ohtsu, "Wide-span optical frequency comb generator for accurate optical frequency difference measurement," Quantum Electronics, IEEE Journal of, vol. 29, no. 10, pp. 2693-2701, 1993.

[8] J. Kakande, A. Bogris, R. Slavik et al., "QPSK phase and amplitude regeneration at 56 Gbaud in a novel idler-free non-gegenerate phase sensitive amplifier.," OFC 2011, Los Angeles, 6-10 Mar 2011 pp. OMT4, 2011. 